\title{
Zwischen Intentionalität und Konvention: Bedeutungskonzepte für kommunikative Handlungen
}

\author{
Gisela Harras
}

\section{Das Problem}

Menschen verwenden Sprache - sprachliche Einheiten wie Wörter, Phrasen und Sätze ihrer Sprache - im allgemeinen dazu, um andere Menschen in irgendeiner Weise zu beeinflussen. Sie wollen mit dem, was sie sagen, oder dadurch, daß sie etwas Bestimmtes sagen, eine bestimmte Wirkung auf ihre Adressaten ausüben. Dies ist offensichtlich ein Truismus. Dennoch tut sich die Linguistik schwer mit ihm: Die Struktur von Sprache als angelegter Sprachfähigkeit und die Struktur von Sprachen als parametrisierte (Grammatiken von) Einzelsprachen können unabhängig von der Möglichkeit ihres Gebrauchs untersucht werden. Dies steht außer Frage, ebenso die triviale Feststellung, daß 'Sprache' an sich nicht instrumentalistisch ist, daß sie also keinen Zweck hat, nicht intentionalistisch ist. Vielmehr sind es einzelne sprachliche Äußerungen, mit denen bestimmte Sprecher Wirkungen hervorbringen wollen, also ganz bestimmte Zwecke und Ziele verfolgen. Wenn man als Linguist oder Linguistin konkrete sprachliche Äußerungen unter dem Gesichtspunkt ihrer tatsächlich hervorgerufenen Wirkungen untersucht - wie dies zum Beispiel in der Diskursanalyse geschieht -, werden solche Untersuchungen nicht mehr erschließen als ein Stück der interaktiven und sozialen Welt der jeweiligen Kommunikationsteilnehmer. Insofern ist Grewendorf (1995, S. 159) zuzustimmen:

»Eine Untersuchung der sozialen und intentionalen Bedingungen für den Gebrauch des sprachlichen Instrumentariums rekonstruiert die externe 'soziale Welt', mit der dieses Instrumentarium korreliert ist, aber sie macht keine Aussagen über dessen innere Strukturierung. Salopp gesagt: Sie liefert für das Instrument 'Gebrauchsanweisungen', aber keine Reparaturanleitung.«

Die Tatsache, daß Untersuchungen konkreter sprachlicher Äußerungen unter dem Gesichtspunkt ihrer kommunikativen Funktion nichts Relevantes über die Struktur von Sprache und Sprachen erschließen können, hat nun bei vielen Linguisten zu dem (Ent-) Schluß geführt, daß Kommunikation als Untersuchungsgegenstand aus dem Bereich der 'eigentlichen' Linguistik zu verbannen sei und 
den Sozialwissenschaften, die sich mit der interaktiven Kompetenz und den entsprechenden Ausprägungen beschäftigen, zugeordnet werden solle (vergleiche zum Beispiel Bierwisch, 1979; Grewendorf, 1995). Eine solche modular begrenzte Arbeitsteilung halte ich für problematisch, nicht nur deswegen, weil sie jedem Laien als völlig unplausibel erscheinen muß, sondern vor allem auch, weil ich es für eine legitime und dringliche Aufgabe der Linguistik halte, die Rolle von Sprache als Organ - mit der Struktur, die sie eben (jeweils) hat - im Zusammenhang mit dem verhaltensregulierenden System, das über dieses Organ verfügt, zu betrachten. Wenn Sprache in dem hier angesprochenen Chomsky-orientierten Verständnis nicht durch ihren Gebrauch als Kommunikationsmittel strukturiert ist, sondern durch eine angeborene genetische Disposition, dann stellt sich die Frage nach den Bedingungen der Möglichkeiten ihres Gebrauchs erst recht! Daß diese Bedingungen nicht in der Struktur der Sprache(n) zu suchen sind, sondern in der Struktur des menschlichen Verhaltens, scheint mir kein ausreichendes Argument für die Notwendigkeit der Selbstgenügsamkeit einer Wissenschaftsdisziplin zu sein.

Soviel dürfte jetzt deutlich sein: Der Ausdruck "Bedeutung" im Titel dieses Beitrags wird hier in erster Linie als Prädikat für bestimmte Formen menschlichen Verhaltens und nicht als Prädikat für sprachliche Einheiten verstanden, im Unterschied zu den Beiträgen von StrauB und Keller (in diesem Band). Und es dürfte wohl auch (hoffentlich) deutlich geworden sein, daß es nicht darum gehen soll, singuläre sprachliche Äußerungen auf ihre jeweils individuellen Sprecherzwecke beziehungsweise deren Hörerinterpretationen hin zu untersuchen, sondern vielmehr um eine Bestimmung dessen, was es heißt, bedeutungsvolle Zeichen (vorwiegend sprachliche, aber nicht ausschließlich) kommunikativ zu gebrauchen.

\section{Die Erklärung menschlichen Verhaltens mit Hilfe geistiger Prädikate}

Der umfänglichste und wohl auch folgenreichste Bestimmungsversuch dessen, was es heißt, daß Menschen mit Zeichen kommunikativ umgehen, stammt bekanntlich von dem englischen Sprachphilosophen Herbert Paul Grice. In seinem bahnbrechenden Aufsatz Meaning (vergleiche Grice, 1989a) werden zunächst, aus der Perspektive eines Interpreten, zwei Arten von "meaning" unterschieden:

(1) "natural meaning", natürliche Bedeutung, die dann vorliegt, wenn man von einem Zustand oder Ereignis auf einen anderen Zustand oder ein anderes Ereignis mit Hilfe der Beziehung der Kausalität schließt; klassische Beispiele hierfür sind: Rauch bedeutet Feuer, schwarze Wolken Regen, Stöhnen Schmerzen, rote Flecken Masern und so weiter. 
(2) "non-natural meaning", nicht-natürliche Bedeutung, die dann vorliegt, wenn wir menschliches Verhalten als einen Versuch interpretieren, uns etwas zu verstehen zu geben; klassische Beispiele hierfür sind: das Winken des Schwimmers bedeutet, daß er Hilfe benötigt; dreimaliges Läuten des Busschaffners bedeutet, daß der Bus voll ist; A's Kopfschütteln bedeutet, daß er anderer Meinung ist als B; A's Äußerung von "Jetzt raus hier!" bedeutet, daß B den Raum verlassen soll; und so weiter. Diese Griceschen Beispiele machen deutlich, daß es sich bei der Bestimmung der nicht-natürlichen Bedeutung wesentlich um die Interpretation von menschlichem Verhalten handelt, wobei Sprache im Spiel sein kann, aber nicht nowendigerweise muß. Dies kann man zunächst als einen Vorteil der Bestimmung sehen: Es soll eine möglichst extensive Explikation kommunikativen Handelns angestrebt werden.

Ein wesentlicher Unterschied zwischen den beiden Fällen von "meaning" besteht nun darin, daß Fälle natürlicher Bedeutung die Bedingung der Faktizität erfüllen müssen, Fälle nicht-natürlicher Bedeutung hingegen nicht (vergleiche dazu Rolf, 1994). Wenn ich sage: "diese Flecken bedeuten Masern", dann lege ich mich darauf fest, da $\beta$ die fleckenbehaftete Person Masern hat. Ich kann nicht sagen: "seine Flecken bedeuten Masern, aber er hat gar keine Masern". Im Unterschied dazu kann man in Fällen nicht-natürlicher Bedeutung das $\mathrm{Zu}$ treffen des zweiten Sachverhalts sehr wohl in Abrede stellen; vergleiche: "dieses dreimalige Läuten bedeutet, daß der Bus voll ist, aber der Bus ist noch gar nicht voll, der Fahrer hat sich geirrt". Darüber hinaus gilt das Unterscheidungskriterium, daß man nicht-natürliche Bedeutung zitieren, in Anführungsstriche setzen kann, zum Beispiel "das Winken des Schwimmers bedeutet: 'Ich brauche Hilfe", was bei natürlicher Bedeutung schlecht möglich ist, vergleiche: "diese schwarzen Wolken bedeuten: 'Es wird regnen'".

Die bei Grice angeführten Beispiele für nicht-natürliche Bedeutung, das haben wir bereits betont, stellen ganz klar Fälle kommunikativer Akte, also Handlungen, dar. Im Unterschied zu den Fällen natürlicher Bedeutungen, in denen es ausreicht, zwei Ereignisse oder Zustände mit Hilfe von Kausalbeziehungen miteinander zu verknüpfen, benötigen wir für die Erklärung von Handlungen weitere Daten. Welcher Art sollen diese sein? Offensichtlich brauchen wir Prädikate für den Handelnden selbst, im weitesten Sinn also Prädikate für geistige Akte. Dies soll durch das folgende Gedankenexperiment, das Grice nachempfunden ist (vergleiche Grice, 1989d), näher erläutert werden.

Angenommen, wir lesen einen Comic, der unter anderem aus den beiden Bildern I und II in Abbildung 1 besteht: Was muß man rekonstruieren, um die beiden Bilder als zusammenhängende Geschichte zu interpretieren? 


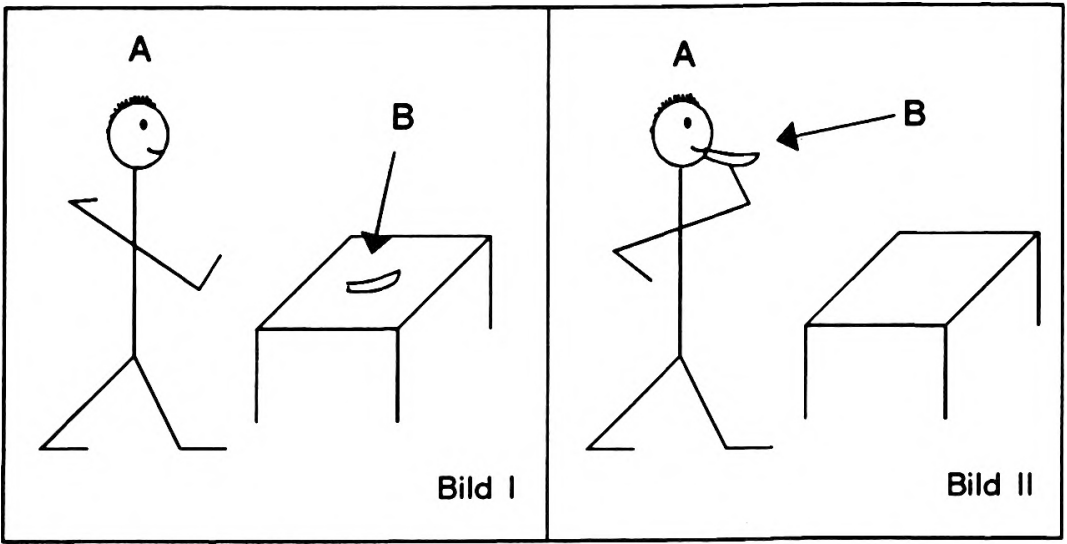

Abbildung 1: Eine kurze Bildergeschichte.

Vermutlich würden wir ihnen zunächst die folgenden Informationen/Daten entnehmen:

- $\quad$ A denkt, daß eine Banane (B) auf dem Tisch liegt;

- $\quad$ A denkt, daß B eßbar und für ihn verfügbar ist;

- A will etwas essen/ist hungrig.

Diese Daten würden wir vermittels einer Art idealisierten Verhaltensmodells in einen Zusammenhang der folgenden Art bringen:

Wenn A glaubt, daß B die Eigenschaften F (eßbar und verfügbar sein) hat und daß Dinge mit der Eigenschaft $F$ zu einer Aktivität $\mathrm{H}$ passen, dann will $\mathrm{A}$ bezüglich $\mathrm{B} \mathrm{H}$ tun.

Wenn man dies nun auf die Prämissen anwendet, erhält man das nächste Stadium, nämlich, daß A B essen will. Dieses Stadium verknüpfen wir vermittels eines weiteren allgemeinen Verhaltensmodell mit dem, was wir auf Bild II sehen. Das Verhaltensmodell kann folgendermaßen formuliert werden:

Wenn eine Person A bezüglich eines Objekts B $H$ tun will und daran nicht in irgendeiner Weise gehindert wird, dann führt $A$ bezüglich $B$ die Aktivität $\mathbf{H}$ aus.

Dies führt zu dem durch Bild II angezeigten Sachverhalt: A ißt B.

Das in den Bildern dargestellte Verhalten von A haben wir mit Hilfe von geistigen Prädikaten wie "denken", "glauben", "wollen" erklärt - wobei es uns in diesem Zusammenhang nicht stört, daß wir epistemische Prädikate wie "denken" und "glauben" in einen Topf werfen. Wichtiger ist, daß wir bei unserem Experiment stillschweigend davon ausgegegangen sind, daß es sich bei $A$ 
um ein rationales Wesen handelt, das um die Korrespondenz seiner internen psychischen Zustände mit den externen Zuständen in der Welt bemüht ist. Gleiches gilt nun auch für kommunikatives Handeln mit der spezifischen Bedingung, da $B$ die externen Zustände, die der Handelnde mit seinen internen Zuständen in Einklang zu bringen versucht, psychische Zustände des jeweiligen Adressaten darstellen. Dies soll wieder durch ein Gedankenexperiment, das ebenfalls bei Grice (1989d) angelegt ist, verdeutlicht werden. Nehmen wir den folgenden Fall, der - um keiner Interpretation Vorschub zu leisten - wieder in einem Bild dargestellt ist (Abbildung 2):

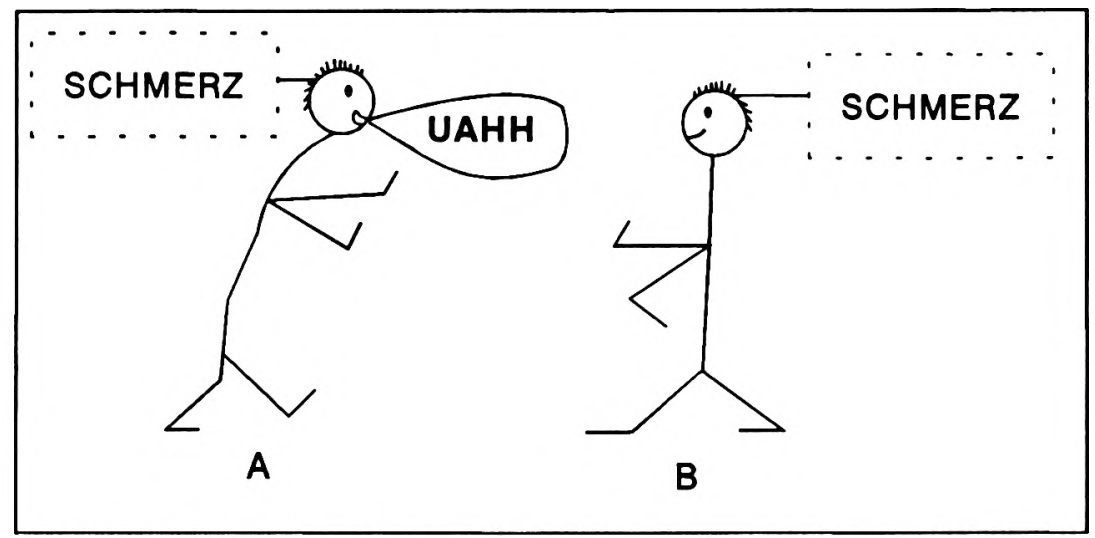

Abbildung 2: Ein Gedankenexperiment.

Wir wissen, daß Stöhnen Schmerzen bedeutet; wir würden also wie Person B zur Überzeugung gelangen, daß A Schmerzen hat. Dies ist ganz klar ein Fall Gricescher natürlicher Bedeutung. Was muß nun hinzukommen, damit die Äußerung der schmerzgeplagten Person als ein Fall nicht-natürlicher Bedeutung gelten kann? Zunächst müßte man sie als eine absichtlich geäußerte erkennen. Dies führt allerdings zu der einigermaßen grotesken Situation, daß die absichtliche Äußerung behandelt werden soll, als sei sie unwillkürlich, unabsichtlich. Die Absicht von A ist von der Art, daß der Rest der Welt glauben soll, er sei in einem Zustand, den das unwillkürliche Zeigen des Verhaltens, das Stöhnen, anzeigen würde. Um sicher zu sein, daß für den Adressaten B das gezeigte Verhalten in der beabsichtigten Weise erkenntlich wird, müßte man des weiteren davon ausgehen können, daß B erkennt, daß A absichtlich eine Form von Verhalten an den Tag legt, dessen unwillkürliche Äußerung Schmerz anzeigt. Diese Erkenntnis würde nun B's Tendenz zu glauben, A habe Schmerzen, gewaltig unterwandern; vermutlich würde B denken, daß A eine witzige Pantomime oder zumindest eine bizarre Komödie aufführt; B würde also an der Ernsthaftigkeit von A's Verhalten zweifeln.

Was muß also der (Beschreibung der) Situation hinzugefügt werden, sozusagen als Mittel gegen die Verwerfung von B's Idee, A habe Schmerzen? Ein 
erster Schritt in diese Richtung würde sicher sein: B erkennt nicht nur, daß A's Verhalten absichtlich ist, sondern $A$ intendiert auch, daB $B$ dies erkennt. Nun sind wir aber schon wieder in einer vertrackten Situation: B soll so etwas denken wie: $\mathrm{Da}$ ist $\mathrm{A}$ mit seiner Simulierung der Schmerzäußerung, zugleich gibt er mir zu verstehen, daß er genau dies tut, nämlich Schmerz simulieren. B kann eigentlich blo $\beta$ zu dem Schluß kommen, daß A eine besondere Art von Verwirrspiel mit ihm veranstalten will. Nun ist $A$ aber gar nicht zum Spielen aufgelegt, sondern er versucht, B ernsthaft glauben zu machen, daß er Schmerzen hat, und das heißt: B soll an die Gegenwart des Zustands bei A glauben, den das geäußerte Verhalten, wenn es unwillkürlich aufträte, natürlicherweise anzeigt. B soll also erkennen, daß A beabsichtigt, da $B$ B erkennt, da $B$ A will, da $\beta$ B glaubt, $A$ habe Schmerzen, und diese Erkenntnis soll $B$ dann auch dazu bringen, daß er tatsächlich glaubt, $A$ habe Schmerzen.

Dieses Gedankenexperiment à la Grice zeigt dreierlei:

(1) Nicht-natürliche Bedeutung - besser: nicht-natürliches Bedeuten oder noch besser: etwas meinen - wird durch die Zuschreibung einer Menge von miteinander zusammenhängenden Sprecherabsichten expliziert.

(2) Entscheidend für den Begriff des Meinens ist nicht die adressatenbezogene Zielorientiertheit des Sprechers, sondern die Art und Weise, wie dieses Ziel zu erreichen versucht werden soll: Wenn die Überzeugung, zu der ein Adressat $\mathbf{H}$ gelangen soll, den Annahmen des Sprechers $\mathbf{S}$ zufolge unabhängig von der Erkenntnis seiner Wirkungsabsicht zustande kommen kann, dann würde man Grice zufolge auch nicht sagen können, $\mathrm{S}$ habe mit $\mathrm{f}$-Tun das-und-das gemeint; man würde in die kausale Erklärungsweise von Bedeutung zurückfallen, das heißt eine Erklärung, die besagt, $\mathrm{da} B \mathrm{f}$ bereits insofern das-und-das bedeutet, als es die Tendenz hat, eine bestimmte Überzeugung hervorzurufen.

(3) Die zugeschriebenen Sprecherabsichten sind offen; das heißt, Fälle von Täuschungen und Simulationen sind ausgeschlossen. Es werden nur diejenigen Sprecherabsichten als Absichten des Meinens angesehen, die offen zu verstehen gegeben werden.

Damit sind wir bei dem angekommen, was zumindest im deutschen Sprachraum als Gricesches Grundmodell (GGM) bekannt geworden ist und die folgenden Explikationen enthält (vergleiche Grice, 1989a, 1989b, 1989c; Meggle, 1981, 1990):

Ein Sprecher $S$ meint ${ }_{N N}$ (NN steht für "non natural") dadurch, daß er f tut, einem Adressaten $\mathrm{H}$ gegenüber etwas dann und nur dann, wenn

(1) S will (mit f-Tun erreichen), daß H eine bestimmte Reaktion r zeigt,

(2) S will, daß H erkennt, daß (1),

(3) $S$ will, daß $H$ aufgrund seiner Erkenntnis von (1) $r$ zeigt. 
(1) ist die primäre Handlungsabsicht des Sprechers, (2) und (3) sind die kommunikativen Absichten, in denen $\mathrm{S} f$ tut, das heißt, eine entsprechende Äußerung macht (vergleiche auch Sperber \& Wilson, 1986). Der Witz des GGM besteht darin, das, was getan wird, in einem systematischen Zusammenhang mit kommunikativen Absichten zu explizieren. Die Reaktion $r$ als primäres Handlungsziel kann in zwei Hinsichten näher präzisiert werden:

(a) $\mathrm{H}$ tut etwas;

(b) $\mathrm{H}$ glaubt etwas.

(b) kann man bezüglich dessen, was $\mathbf{H}$ glauben soll, differenzieren als:

(b1) $\mathrm{H}$ glaubt, daß ein bestimmter Sachverhalt in der Welt besteht;

(b2) $\mathrm{H}$ glaubt, daB sich $\mathrm{S}$ in einem bestimmten psychischen Zustand befindet.

Diese Einteilung würde in etwa der Grobklassifizierung von Sprechakten in Direktive, Assertive und Kommissive entsprechen.

\section{Die Philosophie der Gegenbeispiele}

Das Gricesche Modell hat wie kaum ein anderer theoretischer Entwurf in Sprachphilosphie und Linguistik die Gemüter bewegt und zu Gegenbeispielen angeregt. Ich werde zwei Arten von Gegenargumenten diskutieren:

(a) Das Gricesche Grundmodell ist nicht hinreichend.

(b) Das Gricesche Grundmodell ist zu weitreichend.

\subsection{Das Gricesche Grundmodell ist nicht hinreichend}

Stellvertretend für die Menge der Gegenbeispiele, die demonstrieren sollen, daß das Gricesche Grundmodell nicht hinreichend ist, soll das folgende, von Schiffer (1972) stammende und bei Grice (1989c) selbst diskutierte Beispiel erörtert werden (vergleiche auch Rolf, 1994):

H, ein Mensch, der stets nur aufs Geld aus ist, aber doch auch einen gewissen Stolz besitzt, ist bei $\mathrm{S}$ zu Besuch. $\mathrm{S}$ will den $\mathrm{H}$ loswerden. S wirft daher vor den Augen von $\mathrm{H}$ einen 100-MarkSchein aus dem Fenster. Er will, da B $\mathrm{H}$ die folgenden Überlegungen anstellt: 'S will mich loswerden. Er glaubt, ich werde hinter dem 100-Mark-Schein herrennen. Er will auch, daß ich weiß, daß er will, daß ich gehe (eine derartige Verachtung sprach aus seiner Handlung). Ich werde mich aber nicht so erniedrigen, daß ich 
dem Geldschein nachlaufe. Gut, ich gehe, aber nur, weil er will, daß ich gehe. Wo man mich nicht will, will ich auch nicht bleiben.'

Zunächst scheint es, als seien in diesem Beispielfall alle Bedingungen des Griceschen Grundmodells erfüllt: $S$ tat $f$ (den Geldschein aus dem Fenster werfen) mit der Absicht,

(1) daß H weggeht;

(2) daß H (zumindest zum Teil aufgrund von f-Tun) erkennt, daß S will, daß (1);

(3) daß bei der Erfüllung der Absicht (1) H's Glaube, daß S will, daß (1), für $\mathrm{H}$ ein Grund ist wegzugehen.

Trotzdem hat man intuitiv den Eindruck, daß hier etwas faul ist, oder vornehmer ausgedrückt: $S$ kommuniziert mit $H$ nicht offen über seine Absichten. Dies kann folgendermaßen erklärt werden (vergleiche Rolf, 1994).

Zunächst gilt:

(i) S beabsichtigt, daß $\mathrm{H}$ aufgrund dessen weggeht, daß er erkennt, daß S will, daß er weggeht.

Was S allerdings nicht beabsichtigt, ist, daß

(ii) S will, daß $\mathrm{H}$ erkennt, $\mathrm{da} ß \mathrm{~S}$ will, $\mathrm{da} ß \mathrm{H}$ aus diesem Grund weggehen soll.

H soll lediglich glauben, daß es die Absicht von $\mathrm{S}$ war, $\mathrm{H}$ dadurch loszuwerden, $\mathrm{da} ß$ dieser hinter dem Geld herrennt.

In (i) und (ii) sind H's potentielle Gründe wegzugehen genannt: Der erste Grund, den H - S zufolge - haben soll, ist in der Explikation (2) des Griceschen Modells formuliert, die sich auf diesen Grund beziehende Absicht von S ist in Explikation (3) formuliert. (ii) benennt einen Grund, den $\mathrm{H}-\mathrm{S}$ zufolge - nicht haben soll, das heißt auch, daß das, was in der Explikation (3) formuliert ist, $\mathbf{H}$ - gemä $B$ der Absicht von $S$ - gerade nicht erkennen soll.

Um explikativ zu gewährleisten, daß ein Fall wie der des Schifferschen Geldwerfers aus der Menge der möglichen Beispiele für einen Griceschen Fall des Meinens ausgeschlossen wird, ist es erforderlich, dem Griceschen Grundmodell eine zusätzliche Bedingung hinzuzufügen, die S's Absicht erfaßt, auch die dritte Sprecherabsicht (3) im Grundmodell offen erkennen zu geben, also:

$$
\text { S will, daß H erkennt, daß (3). }
$$

In diesem wie in allen anderen vorgebrachten Gegenbeispielen wird eine Situation entworfen, in der $\mathrm{S}$ immer beabsichtigt, die von ihm gewünschte Reaktion $r$ 
bei $\mathrm{H}$ im Sinn des GGM hervorzurufen, darüber hinaus aber auch immer beabsichtigt, dem $\mathbf{H}$ seine primäre Absicht dadurch zu enthüllen, daß er ihn dazu bringt, ihm eine komplexere Absicht zuzuschreiben, als er tatsächlich hat. Für $\mathrm{H}$ bedeutet dies, daß er - als fiktive Person der Gegenbeispiele - gar nicht in der Lage ist, das Zustandekommen des gewünschten Handlungsziels zu verfolgen: $S$ verbaut ihm sozusagen seine eigene Sicht seines Handlungsentwurfs, und dies ist ganz klar ein Fall von Täuschungsversuch. Um dergleichen aus der Extension der Meinens-Fälle auszuschließen - was ja im Hinblick auf die Forderung nach der Stabilität einer Kommunikationsgemeinschaft wünschenswert wäre -, müßte man S's Absichten so formulieren, daß sie sich darauf richten, von $H$ restlos erkannt zu werden, das heißt, $S$-Absichten müssen als sich selbst enthüllende, selbstbezügliche und selbstreflexive Absichten verstanden werden (vergleiche vor allem Meggle, 1981, 1990). Dies führt in letzter Konsequenz zur Modellierung eines infiniten Regresses von Sprecherabsichten mit entsprechender Verschachtelung von S-Intentionen und H-Erkenntnissen. Einen Ausweg aus dieser Konsequenz haben einige Philosophen darin gesehen, das Gricesche Grundmodell mit einer Anti-Täuschungsklausel der folgenden Art zu versehen:

$\mathrm{S}$ ist mit seinem f-Tun in keiner Weise verheimlichend oder irreführend gegenüber $\mathrm{H}$ in bezug darauf, daß er die Bedingungen (1) bis (3) des Griceschen Mechanismus erfüllt (vergleiche Bennett, 1976; Kemmerling, 1979; Schiffer, 1972, 1987).

Mit einer solchen Antitäuschungsklausel wird allerdings dem ursprünglich deskriptiven Modell von Grice ein präskriptives, kommunikationsethisches Element hinzugefügt, mit dem die subjektiven Sprecherabsichten als für den Hörer verpflichtend zugänglich eingebunden werden sollen.

Grice selbst hat übrigens in einer interessanten Weise auf die Schifferschen (und andere) Gegenbeispiele und ihre Konsequenz reagiert (Grice 1989d). Diese Reaktion soll hier zusammenfassend referiert werden, vor allem auch deshalb, weil sie Aufschluß über den Status des Griceschen Modells gibt. Grice interpretiert die aus den Gegenbeispielen resultierende Konsequenz der unendlichen Verschachtelung von S-Intentionen und H-Erkenntnissen als die unvermeidliche Tendenz zu einem infiniten Regreß: S-Intentionen sind darauf gerichtet, daß sie jeweils die Erkenntnis einer niedrigeren Intention enthalten, das heißt, das Grundschema der Verschachtelung ist das folgende:

$S$ intendiert ${ }_{n}$, daß H erkennt, daß S intendiert ${ }_{n-1}$, daß H ...

Das heißt: Der kognitive Zustand von S, seine Intention, ist, aus seiner eigenen Perspektive betrachtet, immer eine Stufe höher als die S-Intention aus der Perspektive von H. Die Logik der Schifferschen Gegenbeispiele lief nun darauf hinaus, die jeweils höchste S-Intention auch als kognitiven $\mathrm{H}$-Zustand auf dem 
gleichen Rang zu etablieren. Dies ist nun allerdings - wie Grice feststellt - ein logisch unmögliches Unterfangen; das heißt, was wir als kognitiven H-Zustand, also das, was für $\mathbf{H}$ als intendierter Grund oder intendiertes Motiv für die Erfüllung der primären S-Intention dienen soll, auffassen, ist immer eine Stufe hinter dem zurück, was den kognitiven S-Zustand ausmacht, damit eben auch hinter dem, was S selbst als Grund dafür ansieht, warum er will, daß $\mathrm{H}$ eine bestimmte Reaktion $r$ zeigt. Es ist wie mit dem Hasen und dem Igel: Wir fügen immer noch eine Bedingung hinzu, um den Sprecher einzuholen, aber der ist genau dadurch, daß wir dies tun, bereits einen Schritt weiter, das heißt bei der nächsthöheren Intention.

Trotz dieser logischen Unmöglichkeit hält Grice an dem Ideal eines solchen infiniten Regresses fest, obwohl ein solcher psychischer Zustand - wie er selbst sagt - bestenfalls von einem "Rilkeschen Engel« erfüllt werden könnte. Er begründet dies damit, daß es für die Stabilität unserer Kommunikation wünschenswert sei, solche unendlichen Zustände zumindest zu unterstellen. Das Fazit, das Grice aus der Gegenbeispieldebatte zieht, ist zusammengefaßt das folgende:

(1) Ein Sprecher, der mit dem, was er bei einer bestimmten Gelegenheit sagt, meint, daß p, ist in einem optimalen Zustand bezüglich der Tatsache, daß er kommuniziert, daß p.

(2) Der optimale Zustand, in dem der Sprecher eine nicht abzählbare Menge von Intentionen hat, ist nicht realisierbar, so daß er - streng genommen - nicht meint, daß p. Doch befindet er sich in einer Situation, in der es notwendig und im Einzelfall sogar gerechtfertigt ist, daß wir ihn als jemanden ansehen, der diese unerfüllbare Bedingung erfüllt.

Für den Status des Griceschen Mechanismus mit seiner impliziten Forderung nach einem infiniten Regre $B$ von S-Intentionen und H-Erkenntnissen bedeutet dies: Einmal ist er als ein universales Prinzip der Bedingung der Möglichkeit jeglicher menschlicher Kommunikation anzusehen, zum anderen stellt er im Einzelfall jeweils eine Verhaltensmaxime dar, deren Einhaltung durch ein ethisches Prinzip gewährleistet sein sollte.

\subsection{Exkurs: Eine semiotische Reformulierung des Kommunikationsbegriffs}

Der Gricesche Begriff des Meinens, und das heißt auch der Begriff des Kommunizierens, setzt die Existenz bedeutungsvoller Zeichen voraus (vergleiche auch Keller in diesem Band). Posner (1993) hat einen interessanten Versuch unternommen, den Griceschen Begriff semiotisch so zu reformulieren, daß sich das leidige Problem des infiniten Regresses von Intentionen erst gar nicht stellt. Er geht dabei zunächst von fünf elementaren Zeichentypen aus: 
(1) $\quad E(f) \rightarrow E(e)$

Das Auftreten eines Ereignisses $f$ hat kausal das Auftreten eines Ereignisses e zur Folge: Kausalprozeß.

(2) $\quad E(f) \rightarrow T(a, r)$

Das Auftreten eines Ereignisses f löst bei einem reagierenden System, einem Adressaten a ein bestimmtes Verhalten $\mathrm{r}$ aus: Signalprozeß

(3) $\quad E(f) \rightarrow G(a, p)$

Das Auftreten eines Ereignisses f löst bei einem bestimmten Adressaten a einen bestimmten Glauben, daß p, aus: Anzeichenproze $\beta$.

(4) $\quad E(f) \rightarrow G(a, Z(b))$

Das Auftreten eines bestimmten Ereignisses $f$ löst bei einem Adressaten a den Glauben aus, daß sich ein bestimmtes reagierendes System $b$ in einem bestimmten Zustand Z befindet: Ausdrucksproze $\beta$.

(5) $\quad E(f) \rightarrow G(a, I(b(T, b(r)))$

Das Auftreten eines Ereignisses f löst bei einem bestimmten Adressaten a den Glauben aus, daß ein bestimmtes reagierendes Verhaltenssystem b die Absicht hat, r zu tun: Gestenprozeß.

Diese fünf elementaren Zeichentypen sind Empfängerzeichen, das heißt, sie sind strikt aus der Perspektive eines Adressaten bestimmt. Zur Bestimmung von Senderzeichen muß die Zeichendefinition erweitert werden (vergleiche auch Cebulla, 1995). Die Empfängerzeichen sind als Spezialfälle von Kausalprozessen dargestellt; Senderzeichen werden in Erweiterung dazu als Spezialfälle von absichtsvoll herbeigeführten Kausalprozessen dargestellt, das heißt als Handlungen, die so notiert werden:

$$
T(b, f) \& I[b, E(f) \rightarrow E(e)]
$$

f-Tun von b geschieht in der Absicht, mit f-Tun eine Wirkung e herbeizuführen.

Eine weitere Differenzierung des Handlungsbegriffs wird durch die Spezifizierung dessen, worauf sich die Intention des Handelnden richtet, geleistet, so daß vier Typen von Handlungen unterschieden werden können: 
f-Tun von b geschieht in der Absicht, mit f-Tun zu bewirken, daß ein bestimmter Adressat a $r$ tut: Signalisierhandlung.

$T(b, f) \& I[b, E(f) \rightarrow G(a, p)]$

f-Tun von b geschieht in der Absicht, mit f-Tun zu bewirken, da $B$ ein bestimmter Adressat etwas bestimmtes, (daß) p, glaubt: Anzeigehandlung.

$$
T(b, f) \& I[b, E(f) \rightarrow G(a, Z(b))]
$$

f-Tun von b geschieht in der Absicht, mit f-Tun zu bewirken, daß ein bestimmter Adressat a glaubt, daß sich b in einem bestimmten Zustand $Z$ befindet: Ausdruckshandlung.

$$
T(b, f) \& I[b, E(f) \rightarrow G(a, I(b, T(b, r)))]
$$

f-Tun von a geschieht in der Absicht, mit f-Tun zu bewirken, daß ein bestimmter Adressat a glaubt, daß b beabsichtigt, r zu tun: Gestikulierhandlung.

Ein Vergleich der beiden Zeichentypen, das heißt der Empfänger- und Senderzeichen, zeigt, daß letztere komplexer sind als erstere: Senderzeichen in ihrer Eigenschaft als Handlungen enthalten als konstitutive Bestandteile primäre (Empfänger-) Zeichen. Nun sind die hier aufgeführten Handlungstypen für die Bestimmung von Kommunikationsbegriffen auch noch lange nicht hinreichend. Als Beispiel für eine noch relativ einfache kommunikative Handlung soll der folgende, Strawson nachempfundene Fall dienen (vergleiche Strawson, 1974; Cebulla, 1995):

Ein Angestellter spielt gegen seinen Chef Bridge. Er will sich bei seinem Chef lieb Kind machen und will daher bewirken, da $B$ sein Chef denkt, er will, daß sein Chef gewinnt. Er will jedoch nichts tun, was allzu penetrant wäre, wie ihm seine Absicht durch ein direktes Signal zu verstehen geben; eine solche krude Kriecherei würde seinem Chef nun auch wieder nicht passen. Also macht er sich an die Verwirklichung des folgendes Plans: Wenn er gute Karten bekommt, lächelt er - und zwar so, daß sein Lächeln einem spontanen Lächeln zwar sehr ähnlich sieht, aber eben doch nicht so ganz. Dieser Unterschied soll von seinem Chef bemerkt werden, woraufhin dieser dann die folgende Überlegung anstellen soll: 'Das war kein spontanes Lächeln - er tut nur so, als sei er spontan. Das könnte nun einfach ein Versuch sein, mich reinzulegen (vielleicht hat er in Wirklichkeit schlechte Karten). Wir spielen nun aber nicht Poker, sondern Bridge. Und er würde mich, 
seinen Chef, wohl nicht durch eine derartige Ungehörigkeit aufs Kreuz legen wollen. Also hat er vermutlich gute Karten; und da er mich gewinnen lassen will, wird er gehofft haben, daß ich dadurch darauf komme, daß er gute Karten hat, daß ich sein Lächeln für spontan halte: Nun, da dem so ist, werde ich also nicht höher reizen.'

Vor dem Hintergrund der semiotischen Reformulierung von kommunikativen Handlungen kann man den Fall des kriecherischen Angestellten aus der Perspektive seines Adressaten folgendermaßen interpretieren:

(1) Den erfreuten Gesichtsausdruck kann der Adressat als ein Anzeichen deuten, das ihm den Grund dafür liefert, zu glauben, sein Spielpartner habe gute Karten. Dieser Vorgang stellt einen Anzeichenprozeß dar, wie oben unter (3) notiert, und entspricht ganz klar einem Fall von natürlicher Bedeutung à la Grice.

(2) Der Adressat kann den erfreuten Gesichtsausdruck des Angestellten als Auslösung eines Anzeichenprozesses deuten. Er betrachtet den Angestellten als jemanden, der beabsichtigt, ihn mit einem scheinbar spontanen Lächeln zu dem Glauben zu bringen, er habe gute Karten. Der Angestellte führt also eine Anzeigehandlung aus, wie in (8) notiert: $T(b, f) \& I[b, E(f) \rightarrow G(a, p)]$.

(3) Wenn nun - wie in dem obigen Beispielfall angenommen - die Möglichkeit besteht, daß der Adressat an den schauspielerischen Möglichkeiten seines Angestellten zweifelt, dann löst dessen Verhalten keinen Anzeichenprozeß mehr aus, sondern wird von ihm als Anzeichen einer Anzeigehandlung gedeutet werden. Aufgrund der Erkenntnis dieser komplexen Anzeichenprozesse wird der Chef zu dem Glauben kommen, daß sein Angestellter beabsichtigt, eine Anzeigehandlung auszulösen:

$$
E(f) \rightarrow G[a, T(b, f) \& l(b, E(f) \rightarrow G(a, p))]
$$

Das Lächeln bewirkt, daß a glaubt, daß b absichtlich lächelt und damit bewirken will, daß a glaubt, daß b gute Karten hat.

Die Formel (11) ist aus zwei Zeichenprozessen zusammengesetzt: In einen Anzeichenprozeß ist eine Anzeigehandlung eingebettet (siehe Abbildung 3).

Der Vorteil der semiotischen Reformulierung von zeichenvermittelnden Handlungen, das heißt auch von kommunikativen Handlungen, liegt nun ganz offensichtlich darin, aus einem Lexikon elementarer Zeichen, wie sie in (1) bis (10) repräsentiert sind, vermittels einer elementaren Syntax komplexere Zeichenkombinationen gewinnen zu können. Dies wäre vielleicht zunächst nur als eine eher technische Angelegenheit zu betrachten, doch kommen wir auf das Dilemma des Griceschen Mechanismus zurück, das ja in der Gefahr eines infi- 
niten Intentionenregresses bestand. Ein solches Dilemma taucht in dem hier vorgestellten semiotischen Ansatz nicht auf.

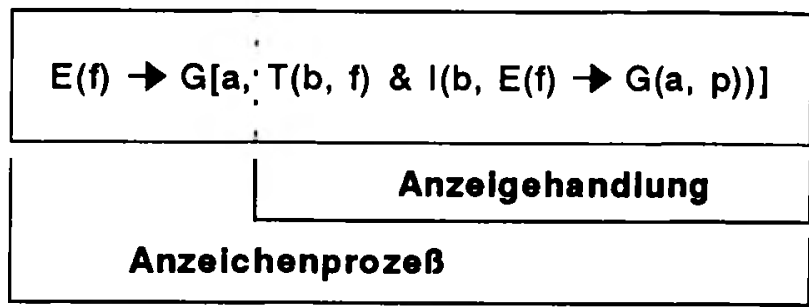

Abbildung 3: Die Zusammensetzung von Formel (11).

Dafür handelt man sich allerdings ein anderes Problem ein, auf das ich gleich zu sprechen kommen werde. Der Witz der semiotischen Konzeption zeichenvermittelnden Handelns besteht darin, dieses als komplexes intentionales Verhalten auf (die Ausbeutung) primäre(r) Zeichenprozesse zu beziehen in der Weise, daß - im Fall komplexer Zeichenprozesse - auch intentionalen Handlungen ein quasi-indexikalischer Charakter zukommt: Mit ihnen zeigen die Zeichenproduzenten an, was sie tun und welche Wirkung sie damit bei ihren Adressaten erzielen wollen. Dazu noch ein Beispiel: In der Geschichte vom kriecherischen Angestellten hat der Chef noch eine vierte Deutungsmöglichkeit, die im übrigen auch diejenige ist, auf die Strawsons Überlegungen zielten (Strawson, 1974). Der Chef kann den in (11) notierten komplexen Anzeichenprozeß als einen vom Angestellten absichtsvoll ausgelösten deuten, das heißt, er kann das folgende Räsonnement anstellen: Indem mein Spielpartner ein spontanes Lächeln zeigt, das doch nicht ganz so spontan erscheinen soll, beabsichtigt er, in mir den Glauben zu erwecken, daß er beabsichtigt, mir die Qualität seiner Karten anzuzeigen. In diesem Fall ist das Verhalten des Angestellten als das Anzeigen einer Anzeigehandlung aufzufassen, die Gesamthandlung zeigt die eingebettete Handlung an:

$$
T(b, f) \& I[b, E(f) \rightarrow G(a, T(b, f) \& I(b, E(f) \rightarrow G(a, p)))]
$$

b zeigt durch sein Lächeln an, daß er beabsichtigt, daß a glaubt, daß er durch sein Lächeln beabsichtigt, daß a glauben soll, daß er gute Karten hat.

Komplexe Zeichenhandlungen unterscheiden sich von elementaren durch unterschiedliche Erfolgsbedingungen (vergleiche Cebulla, 1995; Posner, 1993): Eine elementare Anzeigehandlung, wie sie in (8) notiert ist, ist dann erfolgreich, wenn der beabsichtigte Anzeichenproze $B$ stattfindet, das heißt, wenn der Adressat zu einer bestimmten Überzeugung kommt. Die komplexe Anzeigehandlung (12) ist dann erfolgreich, wenn der Adressat die Absicht, eine Anzei- 
gehandlung auszuführen, versteht. Mit komplexen Zeichenhandlungen wie (12) werden zwei Intentionen miteinander verknüpft: die Intention, eine bestimmte Überzeugung hervorzurufen - dies entspricht der Griceschen primären Intention - und eine Intention, die Art und Weise des Zustandebringens der Adressatenüberzeugung anzuzeigen - dies entspricht der ersten Griceschen kommunikativen Intention. Die zweite Intention hat in der semiotischen Konzeption die Funktion eines Indexes auf die erste Intention: "Die Anzeige einer Anzeigehandlung hat [...] die gleichen Konsequenzen wie sie die tatsächlich vollzogene Anzeigehandlung gehabt hätte.« (Cebulla, 1995, S. 213). Der Erfolg der komplexen Zeichenhandlung bewirkt den Erfolg der elementaren; auf diesen Zusammenhang muß sich der Handelnde verlassen, und in der semiotischen Reformulierung wird dies dadurch repräsentiert, daß ihm diese Überzeugung zugeschrieben wird. Inhaltlich stimmt sie mit der zweite Griceschen kommunikativen Bedingung überein, im Einstellungsmodus ist sie verschieden:

$$
\begin{aligned}
& T(b, f) \& I[b, E(f) \rightarrow G(a, T(b, f) \& I(b, E(f) \rightarrow G(a, p)))] \& \\
& G[b, E(f) \rightarrow G(a, T(b, f) \& I(b, E(f) \rightarrow G(a, p))) \&(E(f) \rightarrow G(a, p))]
\end{aligned}
$$

b zeigt durch $f-T u n$ an, daß er die $A b s i c h t$ hat, daß a glaubt, daß er mit $f-$ Tun beabsichtigt, da $B$ a $p$ glaubt und $b$ glaubt, da $\beta$ f aufgrund dessen, $\mathrm{da} B$ a glaubt, da $\mathrm{B}$ mit $\mathrm{f}-\mathrm{Tun}$ die $\mathrm{Absicht}$ hat, daß a p glaubt, auch tatsächlich bewirkt, daß a p glaubt.

(13) enthält, vereinfacht ausgedrückt:

Bedingung A: Der Handelnde beabsichtigt, daß der Adressat seine Mitteilungsabsicht versteht.

Bedingung B: Der Handelnde beabsichtigt, daß der Adressat seine Mitteilung, die Botschaft, versteht.

Bedingung C: Der Handelnde ist der Überzeugung, daß das Verstehen der Mitteilungsabsicht das Verstehen der Mitteilung bewirkt.

Ist Bedingung A erfüllt, ist die Zeichenhandlung gelungen, das heißt als solche, als Kommunikationsakt zustande gekommen; ist Bedingung $\mathrm{B}$ aufgrund der $\mathrm{Er}$ füllung von A erfüllt, ist die Zeichenhandlung erfolgreich (vergleiche Cebulla, 1995).

Ich habe bereits gesagt, daß in der hier diskutierten Konzeption das Dilemma eines drohenden infiniten Regresses dadurch vermieden wird, daß die Formulierungen, mit denen Zeichenhandlungen repräsentiert werden, Indices enthalten, die als einbettende Zeichenprozesse auf kommunikative Intentionen verweisen. Dadurch, daß diese Indices als spezielle verweisstiftende Prozesse 
ausgezeichnet sind, stellt sich das Problem ihrer Iterierung erst gar nicht. Eine Handlungsbeschreibung der folgenden Art:

Ich zeige an, daß ich anzeige, da $B$ ich anzeige, daß ich mit f-Tun bewirken will, daß q.

sagt - unter Aufwendung einiger Toleranz - nichts anderes als:

Ich zeige an, daß ich mit f-Tun bewirken will, daß $q$.

Ein anderer Vorteil der semiotischen Konzeption liegt nun darin, daß mit ihr deutlich wird, in welchem Maß Kommunikation durch Indirektheit ausgezeichnet ist:

»Der Sender verzichtet darauf, den Adressaten mittels eines der elementaren Zeichenprozesse direkt zu einer bestimmten Reaktion zu bringen; statt dessen verläßt er sich darauf, daß der Adressat so handelt, als ob der elementare ZeichenprozeB stattgefunden hätte, weil er per Anzeigeprozeß von der Intention des Senders unterrichtet wurde, einen solchen elementaren Zeichentyp zu produzieren. Kommunikationsakte zeichnen sich dadurch aus, daß die Wirkungen elementarer Zeichenprozesse durch den Vollzug komplexerer verursacht werden sollen.« (Cebulla, 1995, S. 214.)

Doch leider ist diese eigentlich ganz elegante Bestimmung von Kommunikationsakten nicht unproblematisch. Erinnern wir uns an das Gricesche Grundmodell: Dort war die Bedingung der Vermittlung der beiden Absichten (primäre und kommunikative) im intentionalen Modus repräsentiert, im Unterschied zur semiotischen Reformulierung, in der diese Bedingung im epistemischen Modus erscheint. Intentionen bedürfen nun keiner Rechtfertigung, Überzeugungen sehr wohl. Welcher Art Rechtfertigung könnte man nun für die hier zur Debatte stehende Überzeugung des Handelnden anführen? Posner (1993) und Cebulla (1995) führen sie auf eine Konvention à la Lewis zurück. In bezug auf das Ziel von Verständigung hätten die Handelnden prinzipiell zwei Möglichkeiten: Sie könnten sich nicht-kommunikativ verhalten, das heißt, mit primären Zeichen handeln, oder sie könnten sich kommunikativ verhalten, das heißt, mit komplexen Zeichen handeln. Sie befinden sich also in einer Situation, in der ein koordinatives Gleichgewicht ihrer Handlungen hergestellt werden muB. Wenn sich die Handelnden kooperativ verhalten, werden sie ihr Verhalten mit den Erwartungen, die die übrigen Mitglieder ihrer Gruppe hegen, koordinieren. Sie werden sich also auf eine Verhaltensweise festlegen, und da die Verständigung erheblich erleichtert wird, wenn sie sich möglichst kommunikativ verhalten, werden sie eine entsprechende Konvention ausbilden, die die Erwartungen der Handelnden reguliert und im Einzelfall die Rechtfertigung für 
die Überzeugung der Art des Zustandekommens von beabsichtigten Wirkungen beim Adressaten begründet.

Mit der Koppelung des Griceschen Mechanismus an das Bestehen von Konventionen des kommunikativen Handelns sind zwei mögliche Konsequenzen verbunden:

(a) die Frage, ob dann der Gricesche Intentionalismus überhaupt noch notwendig ist für den Begriff des kommunikativen Handelns. Auf diese Frage komme ich im nächsten Abschnitt zu sprechen, denn sie ist in dieser Form auch von einigen Sprachphilosophen, allen voran von Searle, gestellt und negativ beantwortet worden.

(b) die Frage, inwieweit das Konzept des kommunikativen Handelns erlernt werden muß.

Konventionen müssen - im Unterschied zu genetisch vorgeprägten Strukturprinzipien von Sprache - im Sozialisationsprozeß erlernt werden. In einer Reihe von Experimenten, auf die Olson (1994) verweist, wurden fünfjährige Kinder aufgefordert zu wiederholen, was ein Sprecher mit einer bestimmten Äußerung gesagt und was ein Sprecher mit derselben Äußerung gemeint hat. Die Experimente bezogen sich durchwegs auf Fälle, in denen das, was ein Sprecher gesagt hat, nicht oder nur zu einem Teil mit dem übereinstimmt, was er gemeint hat, also auf Fälle der Verwendung indirekter Sprechakte, metaphorischer oder ironischer Redeweisen. Kinder bis zum Alter von fünf Jahren antworteten unterschiedslos auf beide Fragen mit der Wiedergabe dessen, was wörtlich gesagt wurde, während Kinder ab fünf Jahren durchaus in der Lage waren, ihre Antworten zu differenzieren. Dies legt den Schluß nahe, daß das Konzept des Sagens früher erworben wird als das Konzept des Meinens (vergleiche Olson, 1994). Damit soll nicht behauptet werden, da $\beta$ das Konzept der Intentionalität als solches erlernt werden muß, sondern lediglich das Konzept der kommunikativen Intention: Das, was jemand mit dem, was er sagt (oder tut) meint, ist abhängig davon, was er will, daß sein Adressat glaubt (oder tut); das heißt, es handelt sich um eine Relation zweiter Ordnung. In dieser Komplexität wird der Grund dafür gesehen, daß das Konzept des Meinens später erworben wird als das Konzept des Sagens. Anders gesagt: Im Verlauf der Sozialisation werden Formate des kommunikativen Handelns mit (sprachlichen) Zeichen sukzessive ausgebildet (vergleiche dazu auch Hoffmann in diesem Band).

\subsection{Das Gricesche Grundmodell ist zu weitreichend}

Einer der ersten Kritiker des Griceschen Grundmodells war bekanntlich Searle. Seine Einwände richten sich auf zweierlei: einmal auf die Perspektivik des Grundmodells, die ausschließlich auf den Sprecher festgelegt ist, und zum anderen auf die Bestimmung dessen, was die beabsichtigte Wirkung beim Adres- 
saten sein soll. Der erste Einwand zielt auf die globale Natur des Griceschen Bestimmungsversuchs, der ja so weit gefaßt ist, daß mit ihm alle Fälle kommunikativen Handelns abgedeckt sein sollen, sowohl solche des sprachlichen wie des nicht-sprachlichen kommunikativen Handelns.

Searle verlegt den Schwerpunkt seiner Kritik ausschließlich auf sprachliches Handeln, indem er die Gricesche Fragestellung umdreht: Nicht die Frage, was ein Sprecher mit dem, was er sagt, meint, ist für ihn vordringlich, sondern die Frage nach der Bedeutung der Ausdrücke, mit denen er etwas sagt. Dies macht er an einem berühmt gewordenen Beispiel klar, das - nicht zuletzt wegen seines Ausdrucks der mißverstandenen Deutung der Griceschen Konzeption hier kurz erörtert werden soll (vergleiche Searle, 1969). Searle gibt den folgenden Fall als einen des Griceschen Meinens aus: Ein amerikanischer Soldat, der in italienische Kriegsgefangenschaft geraten ist, will die Italiener glauben machen, er sei ein deutscher Soldat. Zu diesem Zweck sagt er den einzigen deutschen Satz, den er kennt, die Goethe-Zeile: "Kennst du das Land, wo die Zitronen blühn?" Searle fragt angesichts dieses Falls, ob es realistisch sei, davon auszugehen, daß der Amerikaner mit dem Goethe-Zitat im Griceschen Sinn meinen könne, er sei ein deutscher Soldat. Wenn er diese Frage verneint, dann hat er den Ausdruck "meinen" ("to mean") klammheimlich uminterpretiert zu "bedeuten": Natürlich bedeutet der Ausdruck "Kennst du das Land ..." nicht "Ich bin ein deutscher Soldat". Grice hat in einer Replik auf Searle (Grice, 1989c) erwidert, das Beispiel des kriegsgefangenen Amerikaners falle überhaupt nicht unter seinen Begriff des nicht-natürlichen Meinens; allenfalls könne es als ein Fall natürlichen Meinens interpretiert werden: Die Italiener könnten das Sprechen des Gefangenen als ein Anzeichen dafür auffassen, daß er sich als jemand Bestimmtes zu erkennen geben will, oder sie können aufgrund der Tatsache, $\mathrm{da} ß$ es sich um einen deutschen Satz handelt - soviel erkennen sie - schließen, $\mathrm{da} B$ der Sprecher ein deutscher Soldat ist. In diesen Fällen handelt es sich immer um solche der natürlichen Bedeutung (vergleiche dazu auch Rolf, 1994). Semiotisch gesehen stellt der erste Fall ein Anzeichen dar, der zweite das Anzeigen eines Anzeichenprozesses. Innerhalb der in diesem Band dargestellten psychologischen Bedeutungskonzeption würde der Fall des Erkennens, daß der Amerikaner deutsch spricht, als Aktivierung eines Wortmarkenkomplexes gedeutet werden müssen (vergleiche Herrmann, Grabowski, Schweizer \& Graf in diesem Band).

Searle - und mit ihm eine ganze Reihe von Grice-Kritikern (vergleiche Davis, 1992; von Savigny, 1983; Vlach, 1981) - hält die Gricesche kommunikative Intention überhaupt nicht für konstitutiv: Die Bedeutung der Ausdrücke in einer Sprache legen einen jeweiligen Sprecher darauf fest, was er mit diesen Ausdrücken meinen kann, das heißt im wesentlichen auf die Repräsentation eines bestimmten kognitiven Zustands oder einer Einstellung. Wenn jemand zum Beispiel sagt: "Jetzt hol das Bier aus dem Keller!", dann legt er sich vermittels der Bedeutung dieses Ausdrucks auf die Einstellung fest, daß er will, daB. der Adressat das Bier aus dem Keller holt. Seine Sprecherabsicht be- 
schränkt sich darauf, vermittels der Bedeutung des Ausdrucks verstanden zu werden. Aber auch ohne diese Verstehensabsicht kann man mit sprachlichen Ausdrücken etwas meinen, nämlich das Anzeigen der Repräsentation eines bestimmten kognitiven Zustands oder einer Einstellung (vergleiche Searle, 1983). Primär für den Gebrauch der Sprache ist nicht die kommunikative Absicht, sondern die Absicht, eine bestimmte propositionale Einstellung kundzutun.

Diese Reaktion auf Grice geht meines Erachtens von einer grundfalschen Einschätzung aus: Searle versteht Gricesches nicht-natürliches Meinen als einen Akt der willkürlichen Bedeutungsverleihung durch einen Sprecher. Als Kronzeugen für die Unmöglichkeit einer solchen Auffassung zitiert er Wittgenstein, der in seinen Philosophischen Untersuchungen die Suggestivfrage stellt: "Kannst du sagen: 'hier ist es kalt' und meinen: 'hier ist es warm'?" und stillschweigend von der Antwort "Nein" ausgeht. Dies trifft selbstredend dann zu, wenn man die Frage Wittgensteins so versteht: "Kannst du machen, daß das Wort 'kalt' auch 'warm' bedeuten kann?" Diese Frage ist natürlich zu verneinen, während die Frage, ob ein Sprecher im Griceschen Sinn sagen kann "hier ist es kalt" und meinen "hier ist es warm" mit Hinweis auf ironische Redeweisen ohne weiteres bejaht werden kann.

\section{Fazit}

Als Fazit meiner Überlegungen möchte ich eine bündige Antwort auf die Frage nach dem Status eines intentionalistischen Modells kommunikativen Handelns geben. Grice hatte ja eine möglichst extensive Bestimmung geliefert, wobei der Fall des sprachlichen Handelns nur einer unter anderen möglichen Fällen darstellte. Genau dieses Vorgehen der Subsumierung sprachlichen Handelns unter andere Formen des kommunikativen Verhaltens hat die Kritik der Gegner provoziert. Da sprachliche Zeichen, so ihre Argumentation, konventionell für ihren Gebrauch geregelt sind, braucht man den ganzen Intentionalismus nicht! Oberflächlich betrachtet scheinen sie recht zu haben: Wenn jemand meint, was er sagt, das heißt, wenn er eindeutige sprachliche Ausdrücke verwendet, wird der Gricesche Mechanismus zur Erklärung des je konkreten Sprechermeinens mehr oder weniger überflüssig. Diese Argumentation scheint allerdings etwas vorschnell zu sein, denn mit ihr wird stillschweigend die Existenz konventionalisierter Sprachzeichen vorausgesetzt, ohne auf die Frage nach der Möglichkeit ihres Zustandekommens einzugehen, was aber gerade ein Anliegen von Grice gewesen ist, wie das Experiment mit dem schmerzgeplagten Individuum zeigt. Konventionen setzen soziale Praktiken voraus, also Handlungen und damit Intentionen. Diese sind im Gebrauch der konventionalisierten Sprachzeichen sozusagen 'aufgehoben' (vergleiche dazu auch Keller, 1995). Deshalb stellt sich die Frage nach der Intentionalität in all den Fällen nicht, in denen das, was der Sprecher meint, von der Bedeutung seiner verwendeten Ausdrücke determiniert wird. Sie stellt sich allerdings dann, wenn Zweifel bei den Kommunika- 
tionspartnern bezüglich der Verläßlichkeit auf ihr gemeinsames Wissen auftauchen. Dies trifft auf Situationen zu, in denen der Sprecher sprachliche Konventionen ausbeutet, und dies trifft auf Situationen zu, in denen die vom Sprecher gelieferte Information für den Adressaten in irgendeiner Weise unvollständig oder defizitär ist hinsichtlich seiner Verstehensmöglichkeit der Sprecherhandlung. Das heißt, daß das Gricesche Grundmodell letztendlich eine Art Inferenzmodell darstellt zur Gewährleistung der Sprecher-Hörer-Koordination in Fällen, in denen dies aufgrund der semantischen Information allein problematisch wäre.

\section{Literatur}

Bennett, J. (1976). Linguistic behaviour. Cambridge, MA: Cambridge University Press.

Bierwisch, M. (1979). Wörtliche Bedeutung - eine pragmatische Gretchenfrage. In G. Grewendorf (Hrsg.), Sprechakttheorie und Semantik (S. 119-148). Frankfurt/M.: Suhrkamp.

Cebulla, M. (1995). Kommunikationsakte und ihr Gebrauch. In F. Liedtke (Hrsg.), Implikaturen. Grammatische Analysen (S. 210-225). Tübingen: Niemeyer.

Davis, W. (1992). Speaker meaning. Linguistics and Philosophy, 15, 223- 253.

Grewendorf, G. (1995). Sprache als Organ - Sprache als Lebensform. Frankfurt/M.: Suhrkamp.

Grice, H. P. (1989a). Meaning. In H. P. Grice, Studies in the way of words (S. 213-223). Cambridge, MA: Harvard University Press.

Grice, H. P. (1989b). Utterer's meaning and intentions. In H. P. Grice, Studies in the way of words (S. 86-116). Cambridge, MA: Harvard University Press.

Grice, H. P. (1989c). Utterer's meaning, sentence-meaning, and word-meaning. In H. P. Grice, Studies in the way of words (S. 117-137). Cambridge, MA: Harvard University Press. 
Grice, H. P. (1989d). Meaning revisited. In H. P. Grice, Studies in the way of words (S. 283-303). Cambridge, MA: Harvard University Press.

Herrmann, Th., Grabowski, J., Schweizer, K. \& Graf, R. (in diesem Band). Die mentale Repräsentation von Konzepten, Wörtern und Figuren (S. 120-152).

Hoffmann, J. (in diesem Band). Die Genese von Begriffen, Bedeutungen und Wörtem (S. 88-119).

Keller, R. (1995). Zeichentheorie. Tübingen: Francke.

Keller, R. (in diesem Band). Begriff und Bedeutung (S. 47-66).

Kemmerling, A. (1979). Was Grice mit 'Meinen' meint. In G. Grewendorf (Hrsg.), Sprechakttheorie und Semantik (S. 67-118). Frankfurt/M.: Suhrkamp.

Meggle, G. (1981). Grundbegriffe der Kommunikation. Berlin: de Gruyter.

Meggle, G. (1990). Grundlegung einer handlungstheoretischen Semantik. Unveröffentlichtes Manuskript.

Olson, D. R. (1994). The world on paper. The conceptual and cognitive implications of writing and reading. Cambridge: Cambridge University Press.

Posner, R. (1993). Believing, causing, intending. The basis for a hierarchy of sign concepts in the reconstruction of communication. In R Joma, B. v. Heusden \& R. Posner (Hrsg.). Signs, search and communication. Semiotic aspects of Antificial Intelligence (S. 215-270). Berlin: de Gruyter.

Rolf, E. (1994). Sagen und Meinen. Paul Grices Theorie der Konversations-Implikaturen. Opladen: Westdeutscher Verlag.

Savigny, E. von (1983). Zum Begriff der Sprache. Konvention. Bedeutung. Zeichen. Stuttgart: Reclam.

Schiffer, S. (1972). Meaning. Oxford: Oxford University Press.

Schiffer, S. (1987). Remnants of meaning. Cambridge, MA: Cambridge University Press.

Searle, J. R. (1969). Speech acts. An essay in the philosophy of language. Cambridge, MA: Cambridge University Press.

Searle, J. R. (1983). Intentionality. An essay in the philosophy of mind. Cambridge, MA: Cambridge University Press.

Sperber, D. \& Wilson, D. (1986). Relevance. Communication and cognition. Oxford: Oxford University Press.

StrauB, G. (in diesem Band). Wort - Bedeutung - Begriff: Relationen und ihre Geschichte (S. 22-46).

Strawson, P. F. (1974). Intention and convention in speech acts. In J. R. Searle (Hrsg.), The philosophy of language (S. 23-38). Oxford: Oxford University Press.

Vlach, F. (1981). Speaker's meaning. Linguistics and Philosophy, 4, 359-391. 